\title{
miRNA expression profiling of lung adenocarcinomas: correlation with mutational status
}

\author{
Sanja Dacic ${ }^{1}$, Lindsey Kelly ${ }^{1}$, Yongli Shuai ${ }^{2}$ and Marina N Nikiforova ${ }^{1}$ \\ ${ }^{1}$ Department of Pathology, University of Pittsburgh Medical Center, Pittsburgh, PA, USA and \\ ${ }^{2}$ University of Pittsburgh School of Public Health, Pittsburgh, PA, USA
}

\begin{abstract}
MicroRNA (miRNA) expression is deregulated in lung cancer, and some miRNAs are associated with poor prognosis and survival. In this study, we investigated the miRNA expression in lung adenocarcinomas with different oncogenic mutations, including EGFR-positive, KRAS-positive and EGFR/KRAS-negative tumors. The expression of 319 miRNAs was evaluated by Exiqon/Luminex microarray, and expression of individual miRNAs was validated by individual RT-PCR assays (Applied Biosystems). Overall, miRNA expression was similar among three mutationally different groups with most upregulated miRNAs being miR-20a, miR-328, miR-34c and miR-18b and most downregulated miRNAs being miR-32, miR-137 and miR-342. Four miRNAs (miR-155, miR-25, miR-495 and miR-7g) were expressed differently among these tumors. miR-155 was upregulated only in EGFR/KRAS-negative group, miR-25 was upregulated only in EGFR-positive group and miR-495 was upregulated only in KRAS-positive adenocarcinomas. In opposite, let-7g was downregulated in all three groups, with more significant downregulation in EGFR/KRASnegative adenocarcinomas. Principal component analysis (PCA) revealed significant correlation between miRNA expression patterns and somatic mutations. In this study, we demonstrated that despite the similarity in miRNA expression among lung adenocarcinomas with different somatic mutations, some miRNAs showed unique expression patterns, which were in strong correlation with the mutation type, suggesting different carcinogenic pathway for these tumors. These miRNAs can be further explored for their diagnostic and prognostic use. Modern Pathology (2010) 23, 1577-1582; doi:10.1038/modpathol.2010.152; published online 3 September 2010
\end{abstract}

Keywords: EGFR-KRAS; lung adenocarcinoma; miRNA

Lung cancer is the leading cause of cancer deaths in both men and women in the United States. ${ }^{1}$ Over the past two decades, adenocarcinoma has replaced squamous cell carcinoma as the most common subtype of non-small cell lung cancer (NSCLC) in the United States. There is a large amount of data published in literature regarding genetic alterations in lung carcinogenesis. ${ }^{2}$ Alterations in the $p 53$ and $R B / p 16$ pathways are common in all subtypes of lung carcinomas. Somatic mutations in certain tyrosine kinases have emerged as central 'drivers' in lung adenocarcinoma carcinogenesis. Activating mutations of EGFR have been reported in adenocarcinomas responsive to EGFR tyrosine kinase

Correspondence: Dr S Dacic, MD, PhD, Department of PathologyPUH C608, University of Pittsburgh Medical Center, 200 Lothrop St, Pittsburgh, PA 15213, USA.

E-mail: dacics@upmc.edu

Received 8 March 2010; revised 7 May 2010; accepted 10 May 2010; published online 3 September 2010 inhibitors (TKIs). ${ }^{3-5}$ Other mutually exclusive mutations and negative predictors to EGFR-TKI, such as $K R A S$ and BRAF mutations, represent alternative mechanisms of activating the MAP kinase pathway in lung adenocarcinomas. ${ }^{6,7}$

Previously unknown markers, such as noncoding RNA gene products, may be important contributors to lung cancer development as well. MicroRNA (miRNA) is a group of endogenous, small, noncoding RNA that can modulate protein expression by regulating translational efficiency or cleavage of target. They regulate the expression of known tumor suppressor genes and oncogenes. miRNA genes are frequently located at fragile sites, regions of loss of heterozygosity, minimal regions of amplifications or common chromosomal breakpoint regions. They are expressed in a tissue-specific manner, and has been shown that the pattern of expression accurately define the specific cancer types. ${ }^{8-14}$

Prognostic and diagnostic significance of miRNA expression has been reported in several malignancies, 
including chronic lymphocytic leukemia, breast, pancreas, colon and lung carcinomas. ${ }^{15-19}$ Let-7 was the first miRNA reported to be aberrantly expressed in lung carcinomas. ${ }^{20-22}$ It has been found that let-7 miRNA family controls the activity of $R A S$ genes and function as tumor suppressor gene. ${ }^{23}$ Lung tumors with reduced levels of let-7 had significantly increased levels of RAS protein and by some reports poor clinical outcome. In addition, induced overexpression of let-7g in human nonsmall cell lung tumors lead to significant growth reduction and reduced tumor burden. ${ }^{24}$ It was also hypothesized that alterations in let-7 represent an early event in lung adenocarcinoma carcinogenesis. Another miRNAs that correlated with poor survival in lung carcinomas were miR-155, miR-21 and miR-106a. ${ }^{19}$ Upregulation of miR-155 alone was found as independent negative prognostic factor in lung adenocarcinomas. ${ }^{19}$ In addition, the expression of miRNA cluster miR-17-92 is increased in lung cancer, particularly in small cell carcinoma. ${ }^{25}$ The predicted targets include PTEN, RB2 and $c-m y c$, the latest which is frequently amplified/overexpressed in small cell lung carcinoma. ${ }^{26} \mathrm{~A}$ different profiles of miRNA expression was observed in lung carcinomas from never-smokers vs smokers. ${ }^{27}$ Recent studies demonstrated that EGFR may be a functional target of miRNAs. ${ }^{28,29}$ MiRNA-128, which is frequently deleted in lung cancers, directly downregulated EGFR, but did not correlate with EGFR mutation and prognosis. Overall, all these studies suggest that miRNAs can be used as diagnostic and prognostic markers of lung cancer.

Recent studies in thyroid cancer demonstrated correlation between miRNA expression profiles and specific oncogenic mutations, but only one report indicated correlation between the presence of EGFR mutations and miRNA profile in lung adenocarcinomas. ${ }^{30}$ Oncogenic mutations represent a significant event in lung adenocarcinoma development and therapeutic target. In this study, we determined and compared miRNA expression profiles in stage I surgically resected lung adenocarcinomas with different oncogenic mutations. The results may suggest a potential therapeutic strategy targeting specific miRNAs in combination with EGFR-TKI or by itself.

\section{Materials and methods}

\section{Lung Tissue Samples}

Snap-frozen tissue samples from surgically resected lung specimens were obtained from the University of Pittsburgh Health Sciences Tissue Bank. Thirty-four non-neoplastic and neoplastic lung samples were analyzed, including 17 primary stage matched lung adenocarcinomas (five EGFR mutated $(E G F R+)$, six KRAS mutated $(K R A S+)$ and six wild types (wt)).

\section{RNA Isolation}

Total RNA was extracted from surgical specimens using Trizol reagent (Invitrogen, Carlsbad, CA, USA) as previously described. ${ }^{30,31}$ RNA yield and purity were determined using the NanoDrop 1000 spectophotometer (ThermoScientific, Wilmington, DE, USA). RNA integrity was assessed for $1 \mu \mathrm{l}$ of sample using the Agilent 6000 NANO kit for the 2100 Bioanalyzer (Agilent Technologies, Santa Clara, CA, USA) according to the manufacturer's protocol.

\section{miRNA Expression Microarray}

Quantitation of expression of 319 mature miRNA was performed using FlexmiR human microRNA pool, version 8 (Exiqon, Vedbaek, Denmark) and analyzed on Luminex 200 (Austin, TX, USA) according to the manufacturer's instructions. In detail, $2.5 \mu \mathrm{g}$ total RNA was labeled with biotin using the FlexmiR MicroRNA Labeling Kit (Luminex) and subsequently hybridized to beads coated with locked nucleic acid probes complementary to mature miRNA sequences. The system calibration was performed using the xMAP calibration control reagents (Luminex). A blank control set of beads was used to normalize the background value for every individual miRNA. The five synthetic controls and four small nucleolar RNA, snoRNA, normalization controls were used to adjust mean fluorescence intensities between samples and between runs using the average correction factor for these controls, as recommended by the manufacturer. Finally, the miRNA expression in tumor samples was quantitated relatively to normal lung tissue using Luminex IS software v.2.3 (Luminex).

\section{miRNA Expression by Real-Time RT-PCR}

The measurement of the expression levels of individual microRNAs was performed using miRNA sequencespecific primers (Applied Biosystems, Foster City, CA, USA) by the real-time RT-PCR-based detection methodology. Briefly, $10 \mathrm{ng}$ of total RNA was reverse transcribed using High-Capacity cDNA Archive kit (Applied Biosystems) followed by amplification on an ABI 7500 Real-Time PCR System (Applied Biosystems). Small nucleolar RNA RNU44 was used as endogenous control. miRNA expression levels were calculated by relative quantitation using the ABI 7500 Real-Time PCR SDS 1.2 software (Applied Biosystems), and the fold expression changes were determined by $2-\Delta \Delta C_{\mathrm{t}}$ method. ${ }^{30}$ The data were presented as the fold change of miRNA expression in lung tumors relatively to normal lung tissues after normalization to an endogenous control (RNU44).

\section{Statistical Analysis}

Principal component analysis (PCA) was conducted for an unsupervised assessment of the relationship 
between mutation type and the miRNA expression. The Kruskal-Wallis test was also applied to assess the difference in the expression levels of miRNA among mutations. Statistical analyses were performed using SAS version 9.2 (SAS Institute, Cary, NC, USA). A significance level is set at 0.05 and all $P$-values reported are two-sided.

\section{Search for Predicted Target Genes}

Putative miRNA target genes were identified using miRBase (http://microrna.sanger.ac.uk), TargetScan (http://genes.mit.edu/targetscan) (Lewis BP 2003 Cell 115:787-798) ${ }^{32}$ and PicTar (http://pictar.bio. nyu.edu) target prediction programs. ${ }^{33}$

\section{KRAS and EGFR Mutational Analysis}

Tumor targets were manually microdissected from the 4- $\mu$ unstained formalin-fixed paraffin-embedded histological sections. DNA was isolated from each target using the DNeasy tissue kit (Qiagen, Valencia, CA, USA) according to the manufacturer's instructions. For the detection of mutations, DNA was amplified with primers flanking exon 2 of the KRAS gene (forward primer 5'-GGTGAGTTTGTATT AAAAGGTACTGG- $3^{\prime}$ and reverse primer $5^{\prime}$-TCCTG CACCAGTAATATGCA-3'), exon 19 of the EGFR gene (forward primer $5^{\prime}$-CCCAGCAATATCAGCCTT AGGTG-3' and reverse primer 5'-CCACTAGAGCTA GAAAGGGAAAGAC-3') and exon 21 of the EGFR gene (forward primer 5'-CCTCACAGCAGGGTCTTC TC- $3^{\prime}$ and reverse primer $5^{\prime}$-CCTGGTGTCAGGAAA ATGCT- $3^{\prime}$ ). Then, PCR products were sequenced in both sense and antisense directions using the BigDye Terminator v3.1 cycle sequencing kit on ABI 3130 (Applied Biosystems), according to the manufacturer's instructions. The sequences were analyzed using Mutation Surveyor software (SoftGenetics, LLC., State College, PA, USA). Each case was classified as positive or negative for the KRAS and EGFR mutation based on the sequencing results.

\section{Results}

Initially, six lung adenocarcinomas (two positive for EGFR mutation, two positive for KRAS mutation and two EGFR- and KRAS-negative tumors) and corresponding normal tissue samples were analyzed by microarray analysis for expression levels of 319 human mature miRNAs. Overall, miRNA expression was similar among three mutationally different groups, confirming the same histopathologic type of these tumors. Several miRNAs (ie, miR-20a, miR-328, miR-34c and miR-18b) were consistently upregulated with more than 20-fold difference as compared with normal lung tissue (Figure 1). Similarly, miR-32, miR-137 and miR-342 downregulated at more than 20 -fold as compared with

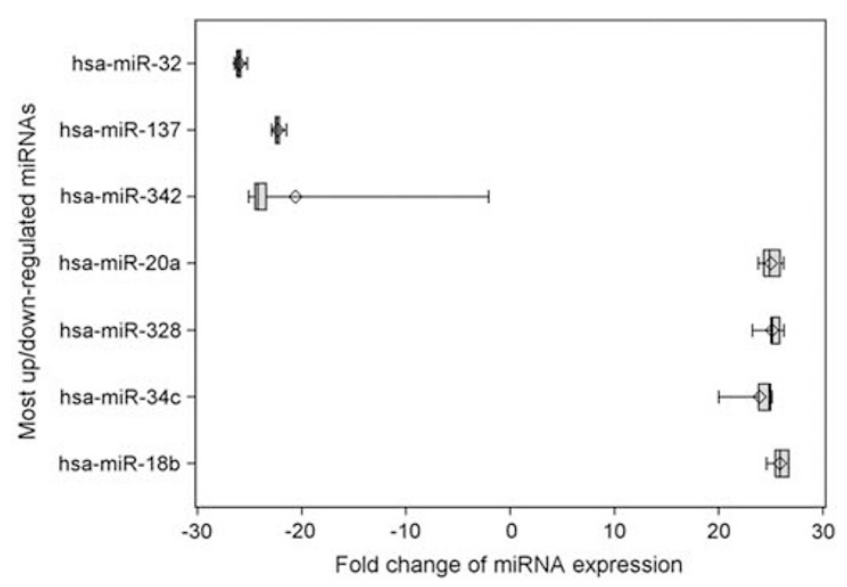

Figure 1 Box plot analysis of the most upregulated and downregulated miRNAs in lung adenocarcinomas.

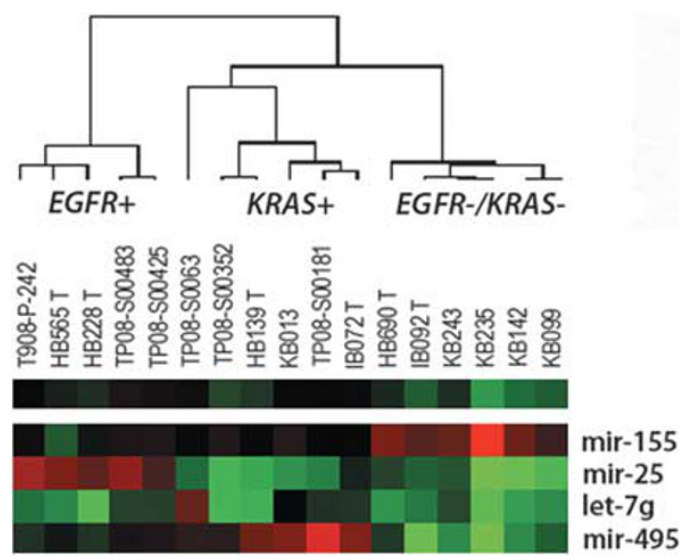

Figure 2 Cluster dendrogram of miRNA expression in lung adenocarcinoma with different oncogenic mutations.

corresponding normal lung tissue (Figure 1). Only one case of adenocarcinoma positive for EGFR mutation did not demonstrate downregulation of miR-342 at such level.

However, several miRNAs were differentially expressed between the mutational groups by microarray analysis. To validate these results, we evaluated expression of each individual miRNA by real-time RT-PCR analysis on six primary studied tumors and on additional 12 adenocarcinoma samples (three EGFR-positive, five KRAS-positive and four EGFR and KRAS-negative tumors). The confirmed dysregulated miRNAs were miR-155, miR-25, miR-495 and miR-7g (Figure 2). Expression of miR-155 and let-7g was of particular interest, as it has been shown that the expression of these miRNAs correlate with a poor prognosis in lung cancer. MiR-155 was upregulated more than twofold in wt adenocarcinomas (EGFR/KRAS negative) and let-7g was downregulated in all three tumor groups, however, more significantly in wt adenocarcinomas as compared with corresponding normal 
Table 1 Differentially expressed miRNAs between EGFR-positive, KRAS-positive and EGFR/KRAS-negative lung adenocarcinomas

\begin{tabular}{lcccc}
\hline miRNA/folds & EGFR+ & KRAS+ & EGFR-/KRAS- & P-value \\
\hline miR-155 & 1.03 & 1.14 & 2.62 & $<0.0005$ \\
miR-25 & 2.37 & 0.44 & 0.38 & 0.0016 \\
miR-495 & 0.97 & 2.43 & 0.37 & $<0.0001$ \\
Let-7g & 0.47 & 0.85 & 0.38 & 0.24
\end{tabular}

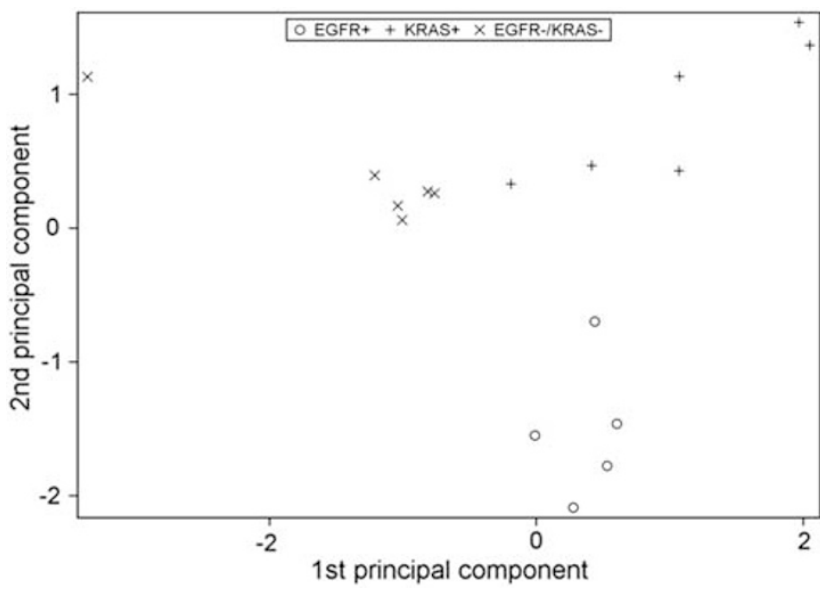

Figure 3 Principle component analysis (PCA) for the assessment relationship between mutation groups and miRNA expression levels.

lung tissue (Table 1). Our study also identified some novel miRNA not previously reported in lung carcinoma: miR-25, which was upregulated more than twofold only in EGFR-positive tumors, and miR-495, which was upregulated more than twofold only in KRAS-positive tumors, as compared with corresponding normal tissue (Table 1). The noted difference in miRNA expression pointed toward the possibility of correlation of miRNA expression with the mutational profile of tumors. To explore this possibility, the unsupervised hierarchical clustering analysis of miRNA expression in the tumors positive for mutations was performed. It demonstrated that EGFR-positive, KRAS-positive and EGFR/KRASnegative adenocarcinomas formed individual clusters (Figure 2). In addition, PCA was used for an unsupervised assessment of the relationship between mutation type and expression levels of the miR-25, miR155, miR-495 and let-7g. It revealed a strong relationship between miRNA expression and mutational status (Figure 3). Similar results were obtained using the Kruskal-Wallis test, which demonstrated significant difference in expression for miR-25, miR-495 and miR-155 $(P=0.0016$ to $P<0.0001)$, whereas the expression of let-7g was not significantly different $(P=0.24)$ (Table 1).

We analyzed the predicted target genes for miR25, miR-495, miR-155 and let-7g (Table 2). The analysis was performed using three publicly available algorithms to predict human miRNA gene
Table 2 Putative target genes of miRNAs dysregulated in lung adenocarcinomas with different oncogenic mutations

\begin{tabular}{|c|c|c|c|}
\hline $\begin{array}{l}\text { Dysregulated } \\
\text { miRNA }\end{array}$ & $\begin{array}{l}\text { miRNA } \\
\text { chromosomal } \\
\text { location }\end{array}$ & $\begin{array}{l}\text { Predicted } \\
\text { gene } \\
\text { name }\end{array}$ & Gene description \\
\hline $\operatorname{miR}-25$ & $7 q 22.1$ & $\begin{array}{l}\text { MAP2K4 } \\
\text { MALAT1 } \\
\text { RAB23 }\end{array}$ & $\begin{array}{l}\text { Mitogen-activated } \\
\text { protein kinase kinase } 4 \\
\text { Metastasis-associated } \\
\text { lung adenocarcinoma } \\
\text { transcript } 1 \\
\text { Member RAS oncogene } \\
\text { family }\end{array}$ \\
\hline miR-495 & $14 q 32.31$ & $\begin{array}{l}\text { CADM1 } \\
\text { (TSLC1) } \\
\text { TCF4 }\end{array}$ & $\begin{array}{l}\text { Tumor suppressor lung } \\
\text { cancer } 1 \\
\text { Transcription factor } 4\end{array}$ \\
\hline miR-155 & $21 \mathrm{q} 21.3$ & $\begin{array}{l}\text { AKAP10 } \\
\text { TCF4 }\end{array}$ & $\begin{array}{l}\text { Homo sapiens A kinase } \\
\text { anchor protein } 10 \\
\text { Transcription factor } 4\end{array}$ \\
\hline let-7g & 3 p21.1 & $\begin{array}{l}\text { NRAS } \\
\text { HMGA2 }\end{array}$ & $\begin{array}{l}\text { Neuroblastoma RAS } \\
\text { viral (v-ras) oncogene } \\
\text { homolog } \\
\text { High mobility group } \\
\text { AT-hook } 2\end{array}$ \\
\hline
\end{tabular}

targets, ie, miRBase, TARGETSCAN and PICTAR. To reduce number of false positives, we listed only putative target genes predicted by at least two of the programs and which were previously implicated in lung carcinogenesis. Table 2 shows target genes putatively regulated by these miRNAs.

\section{Discussion}

Lung adenocarcinoma represents a very heterogenous group of tumor with a large spectrum of morphology, genetics, clinical behavior and variable responses to therapies. Discovery of prognostic and predictive value of EGFR and KRAS somatic mutations in lung adenocarcinomas has changed our understanding of lung carcinogenesis and our perception of lung cancer as a disease. In addition, discovery of differences in miRNA expression between different tumor types provided an insight into lung carcinogenesis and hope for improvement in lung cancer diagnosis, prognosis and potentially new treatment. In this study, we demonstrated that lung adenocarcinomas with different oncogenic mutations have distinct miRNA profiles. As expected, there were many overlapping miRNAs that were upregulated and downregulated regardless of the presence or absence of EGFR and KRAS mutations. This finding correlates with known tissue specificity of miRNA and confirms that all studied tumors were of the same histopathologic type. However, overexpression of miR-25 in EGFR-positive, miR-495 in KRAS-positive and miR-155 in EGFR/KRAS-negative adenocarcinomas may be attributed to the difference in their carcinogenic pathways. 
More interestingly, we found correlation with expression of let-7 and miR-155, two most studied miRNAs in lung cancer, and mutational status. Takamizawa et $a l^{22}$ were first to report reduced expression of let-7 in lung carcinomas associated with shorter survival after potentially curative surgical resection. Yanaihara et $a l^{19}$ also found reduced expression of let-7 family in lung adenocarcinomas. Only let-7a-2 correlated with prognosis by miRNA microarray analysis, but was not statistically significant in the independent set of adenocarcinomas analyzed by real-time RT-PCR analysis. None of these studies compared miRNA profiles with respect to EGFR or KRAS mutations. In study by Inamura et al, ${ }^{21}$ let-7 failed to show prognostic significance in bronchioloalveolar (carcinoma in-situ) and invasive adenocarcinomas. However, mucinous bronchioloalveolar carcinoma showed significantly lower expression than non-mucinous bronchioloalveolar carcinoma. Authors suggested that aberrant let-7 expression most likely represents an early event in lung adenocarcinoma carcinogenesis. A subset of cases was also analyzed for KRAS and EGFR mutations, and no differences were found in let-7 expression between adenocarcinomas with or without EGFR or KRAS mutations. In our study, we found reduced expression of let-7g in all mutational groups with more significant downregulation in tumors negative for EGFR/KRAS mutations. In the same group, we found upregulation of miR-155, another miRNA associated with poor prognosis. Yanaihara et $a l^{19}$ reported a negative prognostic impact of high miR-155 in patients with lung adenocarcinomas. This miRNA was also reported as a prognostic factor in chronic lymphocytic leukemia. ${ }^{15}$ Therefore, on the basis of the reported finding and our results, it is possible to assume that EGFR/ $K R A S$-negative tumors with upregulation of miR-155 and downregulation of let-7g may have the less favorable prognosis as compared with other mutational groups. Our study does not have any survival data, but it would be interesting to further explore prognostic significance of let-7 and miR-155 in stage I wt adenocarcinomas.

Abnormal expression of miR-25 noted in EGFRmutated adenocarcinoma has never been reported in lung carcinoma. Recent analysis of miR-25 expression indicated its role in the regulation of airway smooth muscle cell phenotype by modulating the expression of inflammatory mediators such as RANTES, eotaxin and $T N F \alpha$, as well as genes involved in extracellular matrix turnover and contractile proteins, most notably myosin heavy chain. ${ }^{34}$ Its role in lung cancer is uncertain, but possible role in interaction between malignant epithelial cells and extracellular matrix may be considered. In addition, we report MALAT-1 as one of potential targets of miR-25. Upregulation of this gene was found to be associated with the development of distant metastases in early-stage non-small lung cancer. ${ }^{35,36}$ In our study, miR-25 was upregu- lated only in EGFR-positive group, however, prognostic significance of this finding in our study is uncertain.

Many miRNAs are known to function as oncogenes or tumor suppressors depending on the regulation of their target genes. We identified several interesting genes as predicted targets to differentially expressed miRNAs. A tumor suppressor gene, CADM1 (originally referred as tumor suppressor lung cancer 1 (TSLC1) gene), was a predicted target for miR-495, which is found to be upregulated only in KRAS-positive adenocarcinomas. This gene is known to be inactivated in $44 \%$ of NSCLC, especially in tumors with invasion or metastasis. ${ }^{37}$ The TCF4 gene was identified as target gene for miR-155 and miR-495. This gene is a downstream target of the $\mathrm{Wnt} / \beta$-catenin/TCF pathway, and has been implicated as a marker of metastatic activity in lung cancer. ${ }^{38}$ RAS genes are known predicted targets for let-7. ${ }^{23}$ Finally, the HMGA2 gene is involved in modulation of DNA repair, and overexpression of HMGA2 leads to the promotion of genome instability and tumorigenesis. ${ }^{39,40}$

In conclusion, our results indicate correlation between expression of several miRNAs and somatic mutations in lung adenocarcinomas. These miRNAs may have a role in cancer development and may be associated with more aggressive behavior of some tumor types. With the development of RNAi-based treatments, it might be crucial to learn about specific miRNAs expression and lung adenocarcinoma mutational profile, which may lead to the development of novel therapeutic approaches in lung carcinomas.

\section{Acknowledgement}

We thank Ms Julia Schneider for technical support.

\section{Disclosure/conflict of interest}

The authors declare no conflict of interest.

\section{References}

1 Winer E, Gralow J, Diller L, et al. Clinical cancer advances 2008: major research advances in cancer treatment, prevention, and screening-a report from the American Society of Clinical Oncology. J Clin Oncol 2009;27:812-826.

2 Herbst RS, Heymach JV, Lippman SM. Lung cancer. N Engl J Med 2008;359:1367-1380.

3 Lynch TJ, Bell DW, Sordella R, et al. Activating mutations in the epidermal growth factor receptor underlying responsiveness of non-small-cell lung cancer to gefitinib. N Engl J Med 2004;350:2129-2139.

4 Paez JG, Janne PA, Lee JC, et al. EGFR mutations in lung cancer: correlation with clinical response to gefitinib therapy. Science 2004;304:1497-1500. 
5 Pao W, Miller V, Zakowski M, et al. EGF receptor gene mutations are common in lung cancers from 'never smokers' and are associated with sensitivity of tumors to gefitinib and erlotinib. Proc Natl Acad Sci USA 2004;101:13306-13311.

6 Linardou H, Dahabreh IJ, Kanaloupiti D, et al. Assessment of somatic k-RAS mutations as a mechanism associated with resistance to EGFR-targeted agents: a systematic review and meta-analysis of studies in advanced non-small-cell lung cancer and metastatic colorectal cancer. Lancet Oncol 2008;9:962-972.

7 Pratilas CA, Hanrahan AJ, Halilovic E, et al. Genetic predictors of MEK dependence in non-small cell lung cancer. Cancer Res 2008;68:9375-9383.

8 Calin GA, Croce CM. MicroRNA signatures in human cancers. Nat Rev Cancer 2006;6:857-866.

9 Calin GA, Sevignani C, Dumitru CD, et al. Human microRNA genes are frequently located at fragile sites and genomic regions involved in cancers. Proc Natl Acad Sci USA 2004;101:2999-3004.

$10 \mathrm{Lu} \mathrm{J}$, Getz G, Miska EA, et al. MicroRNA expression profiles classify human cancers. Nature 2005;435: 834-838.

11 Osada H, Takahashi T. MicroRNAs in biological processes and carcinogenesis. Carcinogenesis 2007;28: 2-12.

12 Volinia S, Calin GA, Liu C-G, et al. A microRNA expression signature of human solid tumors defines cancer gene targets. Proc Natl Acad Sci USA 2006;103: 2257-2261.

13 Wijnhoven BP, Michael MZ, Watson DI. MicroRNAs and cancer. Br J Surg 2007;94:23-30.

14 Zhang B, Pan X, Cobb GP, et al. microRNAs as oncogenes and tumor suppressors. Dev Biol 2007;302:1-12.

15 Calin GA, Ferracin M, Cimmino A, et al. A MicroRNA signature associated with prognosis and progression in chronic lymphocytic leukemia. N Engl J Med 2005; 353:1793-1801.

16 Lowery AJ, Miller N, McNeill RE, et al. MicroRNAs as prognostic indicators and therapeutic targets: potential effect on breast cancer management. Clin Cancer Res 2008;14:360-365.

17 Roldo C, Missiaglia E, Hagan JP, et al. MicroRNA expression abnormalities in pancreatic endocrine and acinar tumors are associated with distinctive pathologic features and clinical behavior. J Clin Oncol 2006; 24:4677-4684.

18 Schetter AJ, Leung SY, Sohn JJ, et al. MicroRNA expression profiles associated with prognosis and therapeutic outcome in colon adenocarcinoma. JAMA 2008;299:425-436.

19 Yanaihara N, Caplen N, Bowman E, et al. Unique microRNA molecular profiles in lung cancer diagnosis and prognosis. Cancer Cell 2006;9:189-198.

20 Chin LJ, Ratner E, Leng S, et al. A SNP in a let-7 microRNA complementary site in the KRAS $3^{\prime}$ untranslated region increases non-small cell lung cancer risk. Cancer Res 2008;68:8535-8540.

21 Inamura K, Togashi Y, Nomura K, et al. let-7 microRNA expression is reduced in bronchioloalveolar carcinoma, a non-invasive carcinoma, and is not correlated with prognosis. Lung Cancer 2007;58:392-396.

22 Takamizawa J, Konishi $\mathrm{H}$, Yanagisawa $\mathrm{K}$, et al. Reduced expression of the let-7 microRNAs in human lung cancers in association with shortened postoperative survival. Cancer Res 2004;64:3753-3756.
23 Johnson SM, Grosshans H, Shingara J, et al. RAS is regulated by the let-7 microRNA family. Cell 2005;120: 635-647.

24 Kumar MS, Erkeland SJ, Pester RE, et al. Suppression of non-small cell lung tumor development by the let-7 microRNA family. Proc Natl Acad Sci USA 2008;105: 3903-3908.

25 Hayashita Y, Osada H, Tatematsu Y, et al. A polycistronic microRNA cluster, miR-17-92, is overexpressed in human lung cancers and enhances cell proliferation. Cancer Res 2005;65:9628-9632.

26 O’Donnell KA, Wentzel EA, Zeller KI, et al. c-Mycregulated microRNAs modulate E2F1 expression. Nature 2005;435:839-843.

27 Seike M, Goto A, Okano T, et al. MiR-21 is an EGFRregulated anti-apoptotic factor in lung cancer in never-smokers. Proc Natl Acad Sci USA 2009;106: 12085-12090.

28 Weiss GJ, Bemis LT, Nakajima E, et al. EGFR regulation by microRNA in lung cancer: correlation with clinical response and survival to gefitinib and EGFR expression in cell lines. Ann Oncol 2008;19:1053-1059.

29 Webster RJ, Giles KM, Price KJ, et al. Regulation of epidermal growth factor receptor signaling in human cancer cells by microRNA-7. J Biol Chem 2009;284: 5731-5741.

30 Nikiforova MN, Tseng GC, Steward D, et al. MicroRNA expression profiling of thyroid tumors: biological significance and diagnostic utility. J Clin Endocrinol Metab 2008;93:1600-1608.

31 Nikiforova MN, Caudill CM, Biddinger $\mathrm{P}$, et al. Prevalence of RET/PTC rearrangements in Hashimoto's thyroiditis and papillary thyroid carcinomas. Int J Surg Pathol 2002;10:15-22.

32 Lewis BP, Shih IH, Jones-Rhoades MW, et al. Prediction of mammalian microRNA targets. Cell 2003;115: 787-798.

33 Krek A, Grun D, Poy MN, et al. Combinatorial microRNA target predictions. Nat Genet 2005;37:495-500.

34 Kuhn AR, Schlauch K, Lao R, et al. MicroRNA expression in human airway smooth muscle cells: role of miR-25 in regulation of airway smooth muscle phenotype. Am J Respir Cell Mol Biol 2010; 42:506-513.

35 Ji P, Diederichs S, Wang W, et al. MALAT-1, a novel noncoding RNA, and thymosin beta4 predict metastasis and survival in early-stage non-small cell lung cancer. Oncogene 2003;22:8031-8041.

36 Muller-Tidow $\mathrm{C}$, Diederichs S, Thomas $\mathrm{M}$, et al. Genome-wide screening for prognosis-predicting genes in early-stage non-small-cell lung cancer. Lung Cancer 2004;45(Suppl 2):S145-S150.

37 Goto A, Niki T, Chi-Pin L, et al. Loss of TSLC1 expression in lung adenocarcinoma: relationships with histological subtypes, sex and prognostic significance. Cancer Sci 2005;96:480-486.

38 Vicent S, Luis-Ravelo D, Anton I, et al. A novel lung cancer signature mediates metastatic bone colonization by a dual mechanism. Cancer Res 2008;68: 2275-2285.

39 Di Cello F, Hillion J, Hristov A, et al. HMGA2 participates in transformation in human lung cancer. Mol Cancer Res 2008;6:743-750.

$40 \mathrm{Li}$ AYJ, Boo LM, Wang S-Y, et al. Suppression of nonhomologous end joining repair by overexpression of HMGA2. Cancer Res 2009;69:5699-5706. 